\title{
High-Energy Properties of the Enigmatic Be Star Gamma Cassiopeiae
}

\author{
Chris Shrader*† \\ NASA Goddard Space Flight Center \\ E-mail:Chris.r.shraderenasa.gov
}

\section{S.J. Sturner \& K. Hamaguchi *}

NASA Goddard Space Flight Center

\begin{abstract}
We present the results of a broad-band X-ray study of the enigmatic Be star Gamma Cassiopeiae (herein $\gamma$ Cas) based on observations made with both the INTEGRAL and Suzaku. $\gamma$ Cas has long been recognized as the prototypical example of a small subclass of Be stars with moderately strong X-ray emission dominated by a hot thermal component in the 0.5-12 keV energy range $\left(L_{\mathrm{x}} \approx 10^{32}-10^{33} \mathrm{erg} \mathrm{s}^{-1}\right)$. This places them at the high end of the known luminosity distribution for stellar emission, but several orders of magnitude below typical accretion powered Be X-ray binaries. The INTEGRAL observations spanned an 8 year baseline and represent the deepest measurement to date at energies above $\sim 50 \mathrm{keV}$. We find that the INTEGRAL data are consistent within statistics to a constant intensity source above $20 \mathrm{keV}$, with emission extending up to $\sim 100 \mathrm{keV}$ and that searches for all of the previously reported periodicities of the system at lower energies led to null results. We further find that our combined Suzaku and INTEGRAL spectrum, is fitted extremely well with a thermal plasma emission model with a single absorption component. We found no compelling need for an additional non-thermal high-energy component.
\end{abstract}

10th INTEGRAL Workshop: A Synergistic View of the High-Energy Sky

15-19 September 2014

Annapolis, MD, USA

\footnotetext{
* Speaker.

${ }^{\dagger}$ Also with Universities Space Research Association

${ }^{\ddagger}$ Also with University Of Maryland, Baltimore
} 


\section{INTRODUCTION}

Classical Be stars are B stars whose spectra contain emission lines from $\mathrm{H}$ I while also showing evidence for rapid rotation and equatorially concentrated circumstellar disks [see Rivinius, Carciofi, $\&$ Martayan (2013) for a recent review of Be stars]. $\gamma$ Cas, also known as HD 5394, was the first known Be star (Secchi 1867). It is of spectral type B0.5 IVe and is at a distance of $188 \mathrm{pc}$ with a mass that has been estimated to be in the range 13-18 $\mathrm{M}_{\odot}$ (Zorec et al. 2005). In addition to being the prototypical member of its subclass and one of the brightest, it has been source of extensive study due to its unexpected X-ray properties.

$\gamma$ Cas has previously been studied in the X-ray band and found to have luminosities in the range $10^{32}-10^{33} \mathrm{ergs} \mathrm{s}^{-1}$ and $L_{x} / L_{b o l}>10^{-6}$ (e.g. Smith et al. 2012, 2004). This is significantly larger than the typical $\sim 10^{30} \mathrm{ergs} \mathrm{s}^{-1}$ X-ray luminosity for single O and B stars (Nazé 2009; Oskinova 2005) yet smaller than the X-ray luminosity of Be-neutron star binary systems which during quiescence typically exceed $10^{34} \mathrm{ergs} \mathrm{s}^{-1}$ and during outburst can exceed $\sim 10^{38} \mathrm{ergs} \mathrm{s}^{-1}$ (e.g. Negueruela 1998). There are two classes of models for producing the observed X-rays in $\gamma$ Cas. The currently favored one invokes a magnetic star-circumstellar disk interactions (Robinson et al. 2002) while others involve accretion onto a putative white dwarf binary companion. There are now as many as 10, and possibly 12, similar stars identified in the literature e.g. (Rauw 2013; Motch 2009; Lopes de Oliveira et. al. 2006; Safi-Harb et. al. 2007) sometimes called $\gamma$ Cas analogs. Each are Be stars of similar spectral type (B0.5 - B1.5) with similar X-ray luminosities. Three are apparently in clusters and it has been suggested that they may be blue stragglers which is another possible clue as to their underlying nature.

The soft X-ray spectrum (below $\sim 12 \mathrm{keV}$ ) of $\gamma$ Cas is thermal in nature, being well approximated by optically thin thermal plasma models (Smith et al. 2012, 2004, 1998) and it apparently consists of multiple components (Smith et al. 2012; Lopes de Oliveira et al. 2010). Above $10 \mathrm{keV}$, it is roughly similar in terms of luminosity and spectral energy distribution to hard-X-ray selected cataclysmic variables (CVs) which are primarily intermediate polars (Landi et al. 2009; Revnivtsev et al. 2008). The presence of a white dwarf binary companion has often been suggested as the source of the X-ray emission. However, those objects typically exhibit significant flux and spectral variability above $\sim 20 \mathrm{keV}$ on measurable timescales, e.g. Scaringi et. al. (2010); Landi et al. (2009). Furthermore, there is no compelling evidence in $\gamma$ Cas for the presence of the periodicities that are predominant in CV power density spectra.

Recently, Torrejón et al. (2012) have presented a comprehensive broad-band X-ray spectral study of the $\gamma$-Cas analog HD 110432; also see Lopes de Oliveira et al. (2007). They found that multiple temperature components, possibly including a very hot thermal or non-thermal powerlaw, are needed to adequately fit their data. This result helped motivate us to further explore the brighter and more extensively studied $\gamma$ Cas and in particular to make use of the unprecedented body of highenergy observational data now available from Suzaku and INTEGRAL. In particular, the Cassiopeia sky region received extensive INTEGRAL exposure during the first 8 years of the mission driven largely by nuclear astrophysics studies of the Cas-A remnant.

Previous temporal analysis of the X-ray emission from $\gamma$ Cas has revealed the presence of two components (Smith et al. 1998): 1) a "basal" component that varies on timescales of hours and contributes $\sim 70 \%$ of the X-ray flux, and 2) a "shot" component characterized by fluctuations on 
timescales of seconds to minutes. Henry \& Smith (2012) recently established a revised and more accurate value for the optical signal modulation that they attribute to the stellar rotation period. Their new value is $1.215811 \pm 0.000030$ days, obtained using a comprehensive photometric history spanning a 14-year baseline.

In this paper we consider the hard-X-ray properties of $\gamma$ Cas in the context of this emerging multi-wavelength picture. Our goals are to address the flux history and possible spectral evolution on a range of timescales and interpret our results in the context of current models for the high-energy emission. Of particular interest is the presence, or not, of a non-thermal high-energy component.

\section{OBSERVATIONS AND DATA ANALYSIS}

In this paper we present the results of the accumulated INTEGRAL hard X-ray $/ \gamma$-ray observations of $\gamma$ Cas that span about 8 years. In addition, we have obtained $52 \mathrm{ksec}$ of Suzaku observations with XIS and HXD instruments providing a high-quality spectral determination over the nominal range of $0.1-30 \mathrm{keV}$.

We have analyzed the public data from all spacecraft pointings, or "Science Windows", herein "SCWs", obtained between December 2002 and February 2011 for which $\gamma$ Cas (J2000 $\alpha=14.177$, $\delta=60.717$ ) was within $10^{\circ}$ of the pointing direction. The resulting dataset consisted of 2610 SCWs. Data reduction was performed using the standard OSA 9.0 analysis software package available from the INTEGRAL Science Data Center. Spectral and timing analyses were performed using the XSPEC and XRONOS data analysis packages, respectively.

The Suzaku satellite (Mitsuda et al. 2007) observed $\gamma$ Cas on 2011 July 13. During our Suzaku observations (obsid: 406040010), two sets of instruments were used: the X-ray Imaging Spectrometer (XIS) on the focal plane of the thin-foil X-Ray Telescope (XRT) and the Hard X-ray Detector (HXD). These instruments operate simultaneously on every observation: the net exposures of the XIS and the HXD are $55.4 \mathrm{ksec}$ and $52.3 \mathrm{ksec}$, respectively. A detailed description of the Suzaku spacecraft and mission can be found in the Suzaku technical description ${ }^{1}$.

\section{RESULTS}

\subsection{INTEGRAL Imaging Analysis}

We used the OSA 9.0 software to produce mosaic images of the $\gamma$ Cas region in 3 broad energy bands: $20-40 \mathrm{keV}, 40-60 \mathrm{keV}$, and $60-100 \mathrm{keV}$. We computed the $20-40 \mathrm{keV}$ and $40-60 \mathrm{keV}$ significance maps including all 2601 science windows (Fig. 1). The detection significance using the full dataset is $63.5 \sigma$ and $13.3 \sigma$ in these hard bands. It is thus evident that $\gamma$ Cas has a relatively steep spectrum in this energy range.

Mosaic maps from subsets of the total dataset were inspected to determine a list of sources to include during the spectral and timing analysis steps. This was done to ensure that low duty cycle, transient sources would be accounted for. The datasets consisted of all SCWs from 100 orbit blocks of data. i.e. from orbits 1-100, 101-200, etc. We found 16 sources in the $\sim 20^{0} X 20^{\circ}$ region

\footnotetext{
${ }^{1}$ http://heasarc.nasa.gov/docs/Suzaku/prop_tools/Suzaku_td/
} 


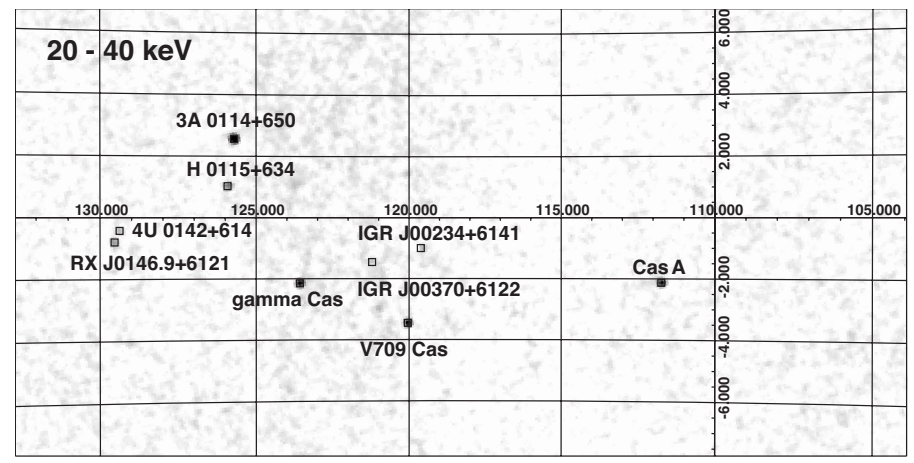

Figure 1: IBIS 20-40 keV band mosaic signifcance map of the $\gamma$ Cas field. It is detected at a significance of $63.5 \sigma$.

around $\gamma$ Cas that we detected at the $5 \sigma$ level or greater during at least one subset. These sources were included in our coded mask solution, leading to the background subtracted image as well as in our and spectral extraction procedure.

\subsection{INTEGRAL Timing Analysis}

As noted earlier, $\gamma$ Cas has been shown to exhibit photometric variations with a 203.55 day periodicity which has been speculatively associated with the orbital motion of a $\sim 1 \mathrm{M}_{\odot}$ companion in a near circular orbit. In addition, a $\sim 1.2$-day modulation has been seen in the B- and V-band photometric monitoring of Henry \& Smith (2012), also see Smith et al. (2006), which is believed to be due to the rotation of the Be star.

The INTEGRAL data set is perfectly suited to produce a long-term lightcurve in order to search for modulations associated with these periodicities and other possible periodicities as well as for stochastic variability. If, for example, the hard-X-ray emission is associated with particle acceleration resulting from a magnetic disk-star interaction scenario, it is not unreasonable to expect the 1.21-day period to emerge, or at least variations at comparable timescales.

We constructed light curves based on single science windows flux determinations in the 20$40 \mathrm{keV}$ and 40-60 keV energy intervals. We then binned the resulting series into sample sizes appropriate for a general variability searches and for period searches, focusing on the published $\mathrm{X}$-ray and optical-UV period determinations.

The flux history of $\gamma$ Cas is shown in Figure 2 where we show the $20-40 \mathrm{keV}, 40-60 \mathrm{keV}$, and ratio light curves rebinned into 48 hour time bins from the intrinsic $\sim 2000$ s single-science window time samples (which defines our maximum time resolution) reflecting the typical length of an INTEGRAL pointing. We also present the hardness-intensity diagram derived from these measurements in the right hand panel. We can see by visual inspection that there is no evidence for large flares but, since some time periods are sparsely sampled, the possibility of low-amplitude flaring cannot be ruled out. We thus formally tested whether light curves, from this time series as well as with several more coarsely binned ones, were consistent with being constant using the chi-square statistic and computing the mean and expected variance for the assumption of a steady source. We did this for different ranges of timescales by binning the data into relatively short (0.5day) and long (30-day) time intervals. The results in all cases were consistent within statistics to 

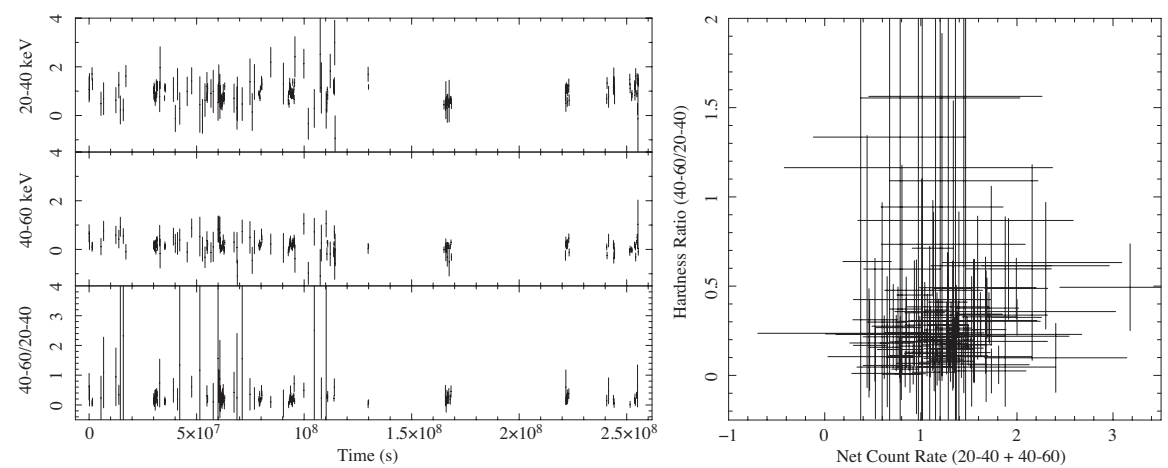

Figure 2: IBIS/ISGRI light curves for $\gamma$ Cas in the 20 - 40 and $40-60 \mathrm{keV}$ energy bands with $1 \sigma$ uncertainties. The light curve consists of 2-day time bins and covers the time period December 2002 to February 2011. The average count rate for this time period is $0.763 \mathrm{cts} / \mathrm{s} \sim 6.0 \mathrm{mCrab}$. For reference, $\mathrm{t}=0$ is MJD $=$ 55755.00035. The right hand panel depicts the hardness intensity space behavior based on these light curve measuremnts.

the light curves being derived from a constant intensity source. For example, for 0.5-day bins using the higher signal-to-noise 20-40 keV band, the observed variance was about one third of the mean and equivalent to the expected variance within about $20 \%$. The corresponding null-hypothesis probability was $10^{-4}$.

Additional variability searches were accomplished using a chi-square test on epoch-folded light curves at and around known periods of the system; specifically the 1.21-day and the 202-day periods associated with Be-star rotation and companion-orbital frequencies. We searched several hundred possible periods on intervals about those values and performed a chi-square test for each case, again testing the constant-source null hypothesis. The results were all negative. For example, we used 20-40-keV band 10-phase light-curve bins to search for 128 possible periods centered on 203.55 days. We found the data to be consistent with having such a 203.55-day periodic component to be only at the $41 \%$ level of confidence.

\subsection{INTEGRAL Spectral Analysis}

We extracted the IBIS/ISGRI hard X-ray time-integrated spectrum for the entire 8-year span using the INTEGRAL OSA tools. Previous analyses of broadband X-ray spectra of $\gamma$ Cas have generally found that the spectra were thermal in nature, typically modeled by optically-thin, thermal plasmas, (Smith et al. 2012, 1998) but in some cases a power-law (or cut-off power-law) model plus a Gaussian line also provided an adequate fit (den Hartog et al. 2006; Smith et al. 1998). We also note the recent analysis of the $\gamma$ Cas analog HD 110432 by Torrejón et al. (2012), where the possible presence of a hard, non-thermal spectral component is identified.

A primary objective here is to characterize the shape of INTEGRAL spectrum and given the apparently flat hard X-ray light curves we focused on the time-integrated dataset. This will offer the best opportunity for the foreseeable future to explore possible deviations from the broad-thermal spectral forms currently established. Towards this end, we first fit the $20-100 \mathrm{keV}$ continuum using a cutoff powerlaw form. Integrated over the $20-100-\mathrm{keV}$ band our best fit model corresponds to a flux of $4.9 \times 10^{-11} \mathrm{ergs} / \mathrm{cm}^{2} / \mathrm{sec}$. In order to best test the hypothesis that an additional hard component, perhaps non-thermal in nature, is superposed on the broad thermal emission we first fitted 
models to the INTEGRAL data alone. We also tried thermal Comptonization spectral forms. In all of these scenarios we found evidence of positive residuals above $\sim 40 \mathrm{keV}$. We thus concluded that the addition of a hard powerlaw component, presumably representing a non-thermal contribution to spectrum, led to a nominal improvement in the quality of the fit. However, an F-Test applied to the two results indicated a probability of only 0.42 that the cutoff powerlaw / thermal Comptonization model fits were improved through the inclusion of the hard tail. Furthermore, that result was strongly dependent on the highest energy channels where the data becomes signal-to-noise limited, and the powerlaw component contributes only about $5 \%$ of the flux.

\subsection{Suzaku Spectral Analysis}

We also performed a separate analysis of the Suzaku spectrum. In the context of the photoionized plasma models, different iron abundances were inferred between the measurements with the K-shell line that originates from $\sim 140 \mathrm{MK}$ plasma and Fe L-shell line complex from the 1-6 MK plasma. Smith et. al. (2004) suggest that these harder X-rays are seen through the dense regions of the circumstellar disk, while most of the X-ray emission is absorbed only by the stellar wind or the outer, less dense regions of the disk. The presence of fluorescence features indeed suggests the presence of gas close to the X-ray emission regions likely associated with the circumstellar medium.

We found that the XIS0+3 and HXD/PIN spectra of $\gamma$ Cas above $0.6 \mathrm{keV}$. The spectrum below $10 \mathrm{keV}$ is similar to those in the earlier Chandra and XMM-Newton observations: hard spectra with three weak emission lines from Hydrogen-like and Helium-like iron and iron fluorescence (Smith et al. 2004; Lopes de Oliveira et al. 2010; Smith et al. 2012). The HXD/PIN spectrum clearly extends up to $\sim 40 \mathrm{keV}$.

\subsection{Combined Spectral Analysis}

The hardness ratios of both the Suzaku 5-9 keV/15-40 keV bands and the INTEGRAL $20-40 \mathrm{keV} / 40-60 \mathrm{keV}$ bands did not show any significant temporal variations. This suggests that the Suzaku and INTEGRAL time-integrated spectra can be jointly fitted by normalizing them at the overlapping energy band between $20-40 \mathrm{keV}$.

For the combined data set we found that the collisionally ionized plasma emission model, apec in the XSPEC package, along with a single absorption term and a Gaussian line component at 6.4 $\mathrm{keV}$ provided an excellent fit to the full data set as was the case with the Suzaku data only (Fig. 3). The inferred plasma temperature was about $15 \mathrm{keV}$, comparable to what others have found for $\gamma$ Cas (e.g. Smith et al. (2004)) and less than that the hot component of HD 110432 of Torrejón et al. (2012). Significantly, the combined fit provides strong evidence that the thermal emission extends up to $\sim 100 \mathrm{keV}$. The inferred luminosity over the $0.1-100 \mathrm{keV}$ band is $\log \left(L_{x}\right)=32.4$. Surprisingly, the continuum extended smoothly into the higher energy INTEGRAL band without requiring a significant instrumental cross-calibration correction. The reduced chi-square statistic for our best overall fit to that model was 1.28 for 1539 degrees of freedom (Fig. 3).

We tried adding a powerlaw component to the model, and found that a similar quality of fit could be obtained. However, that was the case only when the hard powerlaw, index $\Gamma=1.67$, made negligible contribution to the overall flux $(<1 \%)$. Formally, and more dramatically than the 


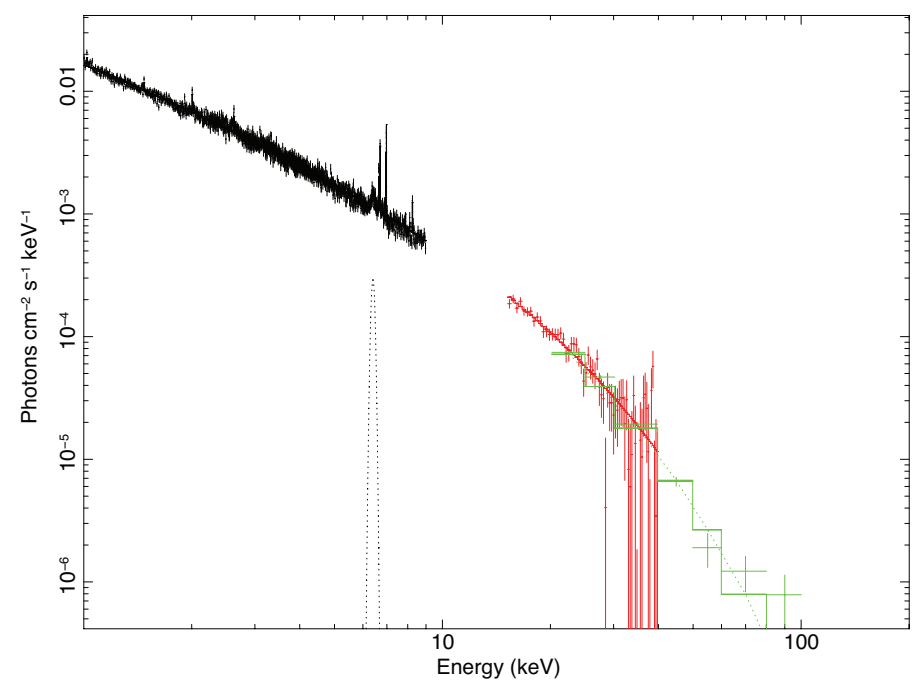

Figure 3: Combined broad-band time, integrated high-energy spectrum of $\gamma$ Cas derived from our analysis of the Suzaku and INTEGRAL datasets described in the text. The model consists of emission from collisionally ionized plasma with temperature of $15.3 \mathrm{keV}$ (based on the "apec" model of the XSPEC software), a Gaussion line feature at $6.4 \mathrm{keV}$ and a single absorption component. Negligible correction for instrumental cross calibration was required. Here we fit the complete data set from $\sim 0.6-100-\mathrm{keV}$. Clearly, the model represents the data extremely well.

INTEGRAL only case, an F-test indicated only a probability of $<1 \%$ that the additional component led to a more accurate representation of the data. Thus, we suggest that a purely thermal plasma emission model is the most realistic representation of the data and the addition of a separate hard component is not justified by our observations.

\section{Discussion and Conclusions}

The ultimate question of how one resolves the long standing $\gamma$ Cas enigma remains but some new clues about this system are slowly emerging. The broadband X-ray emission from the Be stars $\gamma$ Cas can contribute to this endeavor. There is, at this point in time, a fairly broad consensus that the standard Be star X-ray binary model does not provide a plausible description of $\gamma$ Cas. This is in part the case given the lack of orbital modulation and episodic variability as well as the discrepant $\mathrm{X}$-ray luminosity compared to quiescent Be X-ray binaries. Most notable from the perspective of this paper is the nature of the high-energy continuum which in Be X-ray binaries generally displays non-thermal emission components. In transients this can be the case over a range of luminosities; for a recent example see Kühnel et al. (2013).

Scenarios involving a white dwarf companion remain possible but have long been considered problematic and no clear X-ray signatures of binarity have been revealed. One difficulty however with the Be-white dwarf binary scenario for these stars is the problem of forming such a system given the estimated mass ranges for $\gamma$ Cas of 13-18 $\mathrm{M}_{\odot}$ (Zorec et al. 2005). In order for a white dwarf to form in a Be system, it had been long believed white dwarf progenitor must initially be more massive than the Be star progenitor. Evolutionary models by Raguzova (2001) suggest that the maximum initial mass of a white dwarf progenitor in a Be binary system is $10-11 \mathrm{M}_{\odot}$ 
although variations of the traditional shared envelope binary evolution scenarios, involving for example non-conservative mass transfer, could plausibly circumvent this. In the context of this constraint $\gamma$ Cas would then have needed to be initially $\leq 10 \mathrm{M}_{\odot}$ for a white dwarf companion to have formed, but the accretion of $3-8 \mathrm{M}_{\odot}$ or more from the companion to reach its current mass seems unlikely. A putative third body could also alter this picture, but that scenario is highly speculative and improbable.

From purely an observational perspective, while the INTEGRAL CV sample, e.g. (Landi et al. 2009; Revnivtsev et al. 2008), is characterized by a mean (log) luminosity of $L_{20-100 \mathrm{keV}} \simeq 32.5$ which is similar to $\gamma$ Cas, the short-timescale variability characteristics are distinct. The INTEGRAL (and Swift) CV sample is dominated by intermediate polars (IPs) characterized by spin and orbital pulsations and aperiodic fluctuations, none of which is evident here. The CVs typically exhibit softer spectra over that band than what we find for $\gamma$ Cas, on average $\Gamma \simeq 2.9$, however $\mathrm{CV}$ spectra vary significantly in both a phase dependent and an episodic manner.

A very different concept to explain the X-ray emission is the so called "magnetic star-disk interaction" model which was proposed by e.g. Smith et al. (2004); Robinson et al. (2002); Robinson \& Smith (2000); Smith \& Robinson (1999); Smith et al. (1998); also see Smith et al. (2012). In this model it is suggested that many characteristics of the X-ray emission of $\gamma$ Cas can be explained by the dynamical interactions between putative magnetic fields on the star and its circumstellar disk. This seems to be the favored modeling scenario in the current literature, however there are still some puzzling issues; not the least of which is the origin of the magnetic field.

In this scenario short-timescale X-ray variations, as well as correlated UV and optical activity is associated with matter in the proximity of the star being acted upon by magnetic lines of force. This mechanism involves a cyclical magnetic dynamo driven by a Balbus-Hawley instability located within the circumstellar disk (Robinson et al. 2002). According to that model, variations in the magnetic field strength emanating from the star lead to Keplerian shear and turbulent motions within the disk through the magnetorotational instability of Balbus \& Hawley (1991). That mechanism is expected to operate to some extent whenever a magnetic field is embedded in a Keplerian disk. This could result in a positive feedback in which turbulence amplifies the magnetic field, which, in turn, increases the level of turbulence.

The more slowly rotating circumstellar disk drags the field lines causing them on occasion to get entangled. The resulting stretching and reconnection of the field lines leads to particle acceleration, some of which may be directed towards the Be star surface. These streams of plasma then locally heat the photosphere leading to flaring. The heated matter can then expand to lower density regions and continues to glow as the so called "basal" emission component, e.g. (Smith et al. 1998).

The model has been applied to the flux variations that were found on several time scales utilizing optical, UV, and X-ray monitoring of $\gamma$ Cas as detailed in Smith et al. (2004). It was discovered that there was a cyclical variation in both the UV and X-ray data with a 1.21 day period but that the light curves in the two bands were $180^{\circ}$ out of phase (Robinson \& Smith 2000; Smith et al. 1998). Rapid X-ray fluctuations on timescales of seconds to minutes, termed "shots" or "bursts", have also been noted, e.g. Smith et al. (1998); Robinson et al. (2002). The number and amplitude of these shots were found to increase during the UV minimum.

It was proposed that the shots were generated in magnetic structures near the stellar surface and that the 1.21 day period is due to rotation of the star (Robinson \& Smith 2000; Smith et al. 
1998). A possible signature of such models may be magnetically induced flaring, which would likely be pronounced in the hard X-ray band (i.e. above $10 \mathrm{keV}$ ). It would seem highly likely that the high-energy emission would be variable over observable timescales in such scenarios. In the sun for example, where we know for certain that magnetic reconnection events occur they are known to cause particle acceleration leading to rapid and energetic flaring. The disruption and reconfiguration of magnetic field structures associated with the model could also provide a natural explanation for a non-thermal high-energy emission component (although it could also underlie the emergent thermal emission through radiative transfer processes), but we fail to convincingly identify such a component.

To summarize, we have examined more than 8 years of non-uniformly but reasonably wellsampled INTEGRAL observations with fields of view containing $\gamma$ Cas. We do not find significant flux variations in INTEGRAL band, either stochastic or periodic. Our frequency searches included some known periods of the system. We also present our analysis of a $52 \mathrm{ksec}$ Suzaku observation. Temporal analysis of those data considered with the INTEGRAL analysis suggest a decreasing variability with energy. Our multi-instrument spectral analysis suggests a spectral energy distribution which is purely thermal in nature over the broad $\sim 1-100 \mathrm{keV}$ band. While this result is generally consistent with the favored magnetic field - circumstellar disk interaction model it potentially imposes new constraints as the magnetic reconnection mechanism that model invokes could quite possibly entail both high-energy flaring and particle acceleration leading to non-thermal emission.

\section{References}

den Hartog, P. R., Hermsen, W., Kuiper, L., Vink, J., in't Zand, J. J. M. and Collmar, W., 2006, A\&A, 451, 587

Henry, G. W., \& Smith, M. A. 2012, ApJ, 760, 10

Kühnel, M., Müller, S., Kreykenbohm, I., Fürst, F., Pottschmidt, K., Rothschild, R. E., Caballero, I., Grinberg, V., Schönherr, G., Shrader, C., Klochkov, D., Staubert, R., Ferrigno, C., Torrejón, J.-M., Martínez-Núñez, S. \& Wilms, J., 2013, A\&A, 555, 95

Landi, R., Bassani, L., Dean, A. J., Bird, A. J., Fiocchi, M., Bazzano, A., Nousek, J. A. and Osborne, J. P., 2009, MNRAS, 392, 630

Lopes de Oliveira, R., Motch, C., Haberl, F., Negueruela, I. \& Janot-Pacheco, E., A\&A, 2006, 454, 265

Lopes de Oliveira, R., Motch, C., Smith, M. A., Negueruela, I., \& Torrejón, J. M. 2007, A\&A, 474, 983

Lopes de Oliveira, R., Smith, M. A., \& Motch, C. 2010, A\&A, 512, A22

Mitsuda, K., \& et al. 2007, PASJ, 59, 1

Motch, C., Lopes de Oliveira, R., Negueruela, I., Haberl, F. \& Janot-Pacheco, E., 2007, 361, 117, Ed. Okazaki, A. T., Owocki, S. P., \& Stefl, S. 
Nazé, Y., A\&A, 2009, 506, 1055

Negueruela, I. 1998, A\&A, 338, 505

Oskinova, L. M., 2005, MNRAS, 361, 679

Raguzova, N. V. 2001, A\&A, 367, 848

Rauw, G., Nazé, Y., Spano, M., Morel, T. and ud-Doula, A., 2013, A\&A, 555, L9

Rivinius, T., Carciofi, A. C. and Martayan, C., 2013, A\&Ar, 21, 69.

Revnivtsev, M., Sazonov, S., Krivonos, R., Ritter, H., \& Sunyaev, R. 2008, A\&A, 489, 1121

Robinson, R. D., \& Smith, M. A. 2000, ApJ, 540, 474

Robinson, R. D., Smith, M. A., \& Henry, G. W. 2002, ApJ, 575, 435

Safi-Harb, S., Ribó, M., Butt, Y., Matheson, H., Negueruela, I., Lu, F., Jia, S., \& Chen, Y., ApJ, 2007, 659, 407

Scaringi, S. et al., 2010, MNRAS, 401, 2207

Secchi, A. 1867, Astronomische Nachrichten, 68, 63

Smith, M. A., Cohen, D. H., Gu, M. F., et al. 2004, ApJ, 600, 972

Smith, M. A. \& Robinson, R. D. 1999, ApJ, 517, 866

Smith, M. A., Robinson, R. D., \& Corbet, R. H. D. 1998a, ApJ, 503, 877

Smith, M. A., Henry, G. W., \& Vishniac, E. 2006, ApJ, 647, 1375

Smith, M. A., Lopes de Oliveira, R., \& Motch, C. 2012a, ApJ, 755, 64

Smith, M. A., et al., 2012, A\&A, 540, A53

Torrejón, J. M., Schulz, N. S., \& Nowak, M. A., 2012, ApJ, 750, 75

Zorec, J., Frémat, Y., \& Cidale, L. 2005, A\&A, 441, 235 